\title{
Astronomical quantum optics with Extremely Large Telescopes
}

\author{
D. Dravins ${ }^{1}$, C. Barbieri ${ }^{2}$, R. A. E. Fosbury ${ }^{3}$, \\ G. Naletto ${ }^{4}$, R. Nilsson ${ }^{5}$, T. Occhipinti ${ }^{6}$, \\ F. Tamburini ${ }^{7}$, H. Uthas ${ }^{8}$ and L. Zampieri ${ }^{9}$ \\ ${ }^{1}$ Lund Observatory, Lund, Sweden, email: dainis@astro.lu.se \\ ${ }^{2}$ Department of Astronomy, University of Padova, Italy, email: cesare.barbieri@unipd.it \\ ${ }^{3}$ ST-ECF, Garching bei München, Germany, email: rfosbury@eso.org \\ ${ }^{4}$ Department of Information Engineering, Univ. of Padova, Italy, email: naletto@dei.unipd.it \\ ${ }^{5}$ Lund Observatory, Lund, Sweden, email: ricky@astro.lu.se \\ ${ }^{6}$ Dept. of Inform. Engineering, Univ. of Padova, Italy, email: tommaso.occhipinti@dei.unipd.it \\ ${ }^{7}$ Department of Astronomy, University of Padova, Italy, email: tamburini@pd.astro.it \\ ${ }^{8}$ Lund Observatory, Lund, Sweden, email: helena@astro.lu.se \\ ${ }^{9} \mathrm{INAF}$ - Astronomical Observatory of Padova, Italy, email: zampieri@pd.astro.it
}

\begin{abstract}
Modern optics focuses on photonics and quantum optics, studying individual photons and statistics of photon streams. Those can be complex and carry information beyond that recorded by imaging, spectroscopy, polarimetry or interferometry. Since [almost] all astronomy is based upon the interpretation of subtleties in the light from astronomical sources, quantum optics has the potential of becoming another information channel from the Universe. The observability of quantum statistics increases rapidly with telescope size making photonic astronomy very timely in an era of very large telescopes.
\end{abstract}

Keywords. instrumentation: photometers - techniques: spectroscopic - methods: data analysis — radiation mechanisms: nonthermal — methods: statistical — telescopes

\section{Introduction}

For centuries, optical astronomy has developed along with optical physics: Galileo's telescope and Fraunhofer's spectroscope were immediately applied to astronomical problems. The basic physical principles of such imaging and spectroscopic instruments have been applied in the design of cameras and spectrometers for all wavelength ranges.

The frontiers of $21^{\text {st }}$ century optical physics have now moved toward photonics and quantum optics, studying individual photons and photon streams. Those can be complex, carrying information beyond imaging, spectroscopy, or polarimetry. Since [almost] all astronomy is based upon the interpretation of subtleties in the light from astronomical sources, quantum optics appears to have the potential of becoming another information channel from the Universe. What astronomy could then be possible with quantum optics?

\section{What is not observed in optical astronomy?}

Figure 1 illustrates a type of problem often encountered in astrophysics: Light from various sources has been created through different (but typically unknown) physical processes: thermal radiation, stimulated emission, synchrotron radiation, etc. Now, assume one is observing these sources through 'filters', adjusted so that all sources have the same size and shape, same intensity, same spectrum and the same polarization. How can one then tell the difference when observing the sources from a great distance? 


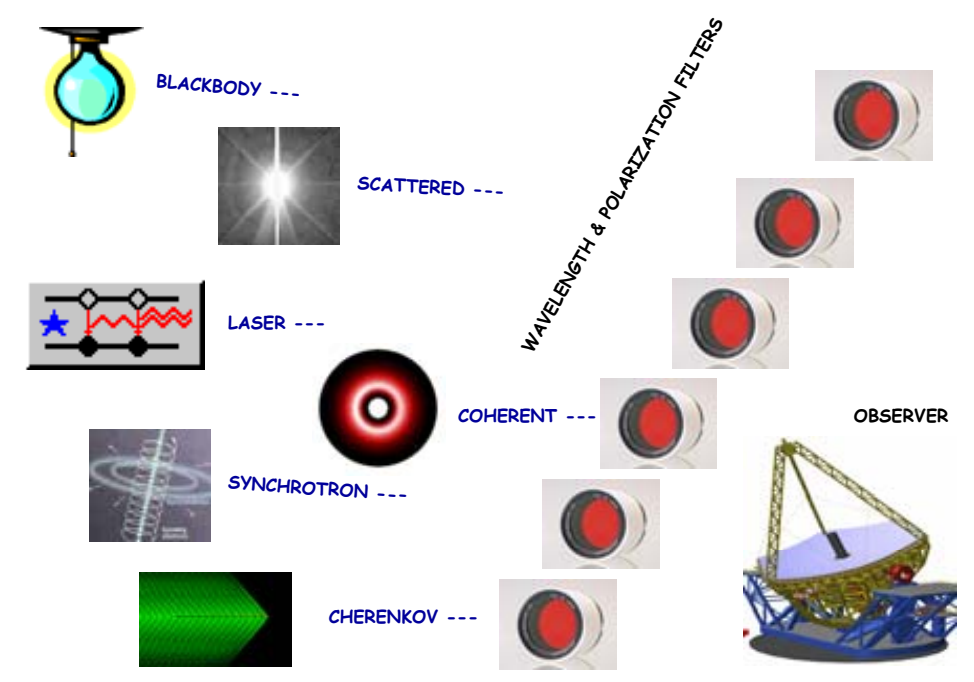

Figure 1. An observational problem: Various sources emit light created through different processes. If spatial and spectral filters give to all sources the same apparent size, same intensity, same spectrum and the same polarization, how can one then tell the difference?

For sources as defined, it is not possible to segregate them using any classical astronomical instrument. Cameras would record the same spatial image and any spectrometer would find the same spectrum. Still, the light from those sources can be different.

Identical images are produced by photons arriving from identical directions, and identical spectra by similar distributions of photon energies. However, a photon stream has further degrees of freedom, such as the temporal statistics of photon arrival times, giving a measure of ordering within the photon-stream and its possible deviations from 'randomness'. Such properties are reflected in the second- (and higher-) order coherence of light, observable as correlations between pairs (or a greater number) of photons.

The stellar intensity interferometer, used to deduce stellar sizes (Hanbury Brown 1974), would today be considered a quantum-optical instrument. The second-order coherence of light was measured from the correlation of intensity fluctuations between two telescopes. However, its functioning implicitly assumes the photon statistics of starlight to correspond to the Bose-Einstein distribution characteristic for thermodynamic equilibrium as from a black-body radiator. A hypothetical star shining with idealized laser light would produce no intensity fluctuations and the instrument would not function.

\section{Ultimate information content of light}

Higher-order coherence of light may convey information about the physics of light emission (e.g., stimulated emission as in a laser) or propagation (e.g., whether photons reach us directly from the source or have undergone scattering on their way: Bachor 1998, Dravins 1994, Loudon 1980, 2000).

The effects become fully visible over timescales equal to the [first-order] temporal coherence time. For astronomically realistic passbands (1nm, say), this is on the order of picoseconds, much shorter than practical photometric resolutions. On more realistic nanosecond scales, the effects are diluted but still measurable, as demonstrated already years ago by the intensity interferometer, the [so far] only astronomical instrument that studied the second-order coherence of light. 


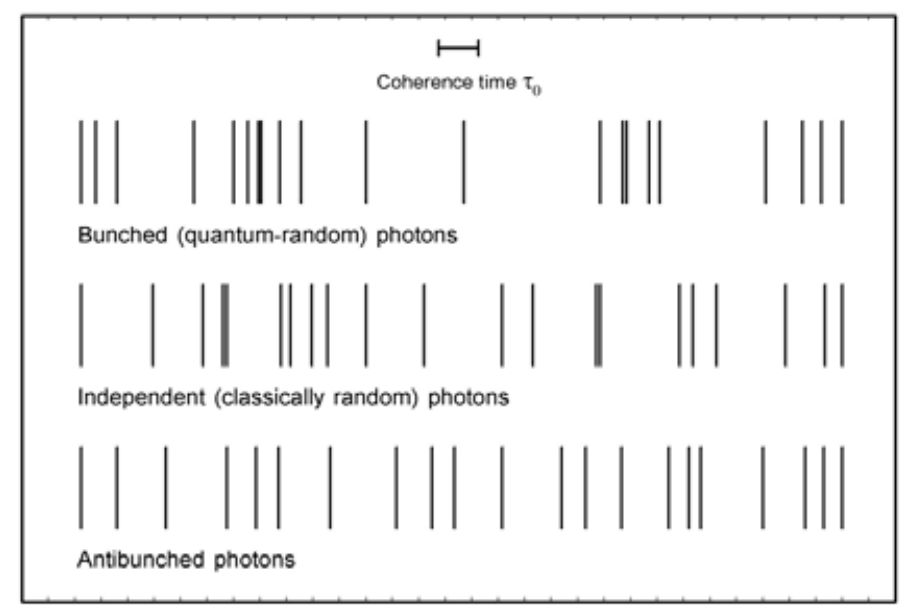

Photon arrival time

Figure 2. Statistics of photon arrival times in light beams with different entropies (different degrees or 'ordering'). The statistics can be 'random', as in maximum-entropy black-body radiation (Bose-Einstein distribution with a certain 'bunching' in time), or may be quite different if the radiation deviates from thermodynamic equilibrium. (Adapted from Loudon 1980, 2000)

\section{Instrumentation for extremely large telescopes}

As part of the instrument design studies for OWL, ESO's planned Overwhelmingly Large Telescope, an instrument for the highest time resolution, QuantEYE, was designed (Dravins et al. 2005, 2006, Barbieri et al. 2006). It is to explore astrophysical variability on microsecond and nanosecond scales, studying a host of astrophysical phenomena in this yet unexplored parameter domain. Those include: millisecond pulsars; variability close to black holes; surface convection on white dwarfs; non-radial oscillation spectra in neutron stars; fine structure on neutron-star surfaces; photon-gas bubbles in accretion flows; and possible free-electron lasers in the magnetic fields around magnetars. Besides such applications in high-speed astrophysics, the aim is to reach timescales sufficiently short to reveal the quantum-optical statistics of photon arrival times.

Using concepts related to the [spatial] intensity interferometer, analogous 'interferometry' in the time domain ('photon-correlation spectroscopy', also used in many laboratory experiments) will enable QuantEYE to reach a spectral resolution $\lambda / \Delta \lambda \approx 100,000,000$, as required to resolve known optical laser emission around the luminous object Eta Carinae. Theoretically expected emission linewidths there are on the order $\approx 10 \mathrm{MHz}$ (Johansson \& Letokhov 2004, 2005), which can be resolved by photon-correlation spectroscopy with time resolution $\Delta \mathrm{t} \approx 100 \mathrm{~ns}$. This may open up optical astronomy to extremely high spectral resolutions, although - analogous to spatial information from intensity interferometry - photon correlation spectroscopy does not reconstruct the full shape of the source spectrum, but 'only' gives linewidth information.

\subsection{Further quantum-optical measures}

Laboratory and theoretical studies in quantum optics have demonstrated that even individual photons of some specific wavelength and arriving from a given direction may carry additional information. In addition to their 'regular' angular momentum (associated with circular polarization), individual photons may carry various amounts of POAM, photon-orbital-angular-momentum (e.g. Padgett et al. 2004). While the existence of astrophysical POAM sources is still unclear (Harwit 2003), POAM manipulation at the 


\begin{tabular}{|c|c|c|c|}
\hline Telescope diameter & Intensity $\langle I\rangle$ & $\begin{array}{l}\text { Second-order } \\
\text { correlation }\langle I 2\rangle\end{array}$ & $\begin{array}{c}\text { Fourth-order photon } \\
\text { statistics }\langle I \text { i }\rangle\end{array}$ \\
\hline $3.6 \mathrm{~m}$ & 1 & 1 & 1 \\
\hline $8.2 \mathrm{~m}$ & 5 & 27 & 720 \\
\hline $4 \times 8.2 \mathrm{~m}$ & 21 & 430 & 185,000 \\
\hline $50 \mathrm{~m}$ & 193 & 37,000 & $1,385,000,000$ \\
\hline $100 \mathrm{~m}$ & 770 & 595,000 & $355,000,000,000$ \\
\hline
\end{tabular}

Figure 3. Light-curves become useless for resolutions below microseconds where typical time intervals between successive photons may even be longer than the time resolution. Instead studies have to be of the statistics of the arriving photon stream, such as its correlations or power spectra. All such statistical functions depend on [at least] the second power of the source intensity. The table compares the observed signal $(I)$, its square and fourth powers for telescopes of different size. The signal for classical quantities increases with the intensity $I$, the signal in power spectra as $I^{2}$ and that of four-photon correlations as $I^{4}$. This very steep dependence makes the largest telescopes enormously more sensitive for high-speed astrophysics and quantum optics.

telescope may offer possibilities for high-contrast imaging inside an 'optical vortex', with a possible application in exoplanet imaging (Swartzlander 2001).

\section{Acknowledgements}

The work in Lund was supported by the Swedish Research Council, the Royal Physiographical Society in Lund, and the European Southern Observatory. The work in Padova was supported by the University of Padova, INAF and the European Southern Observatory. At Lund Observatory we had valuable discussions with, in particular, Daniel Faria, Lennart Lindegren and Hans-Günter Ludwig.

\section{References}

Bachor, H. A. 1998, A Guide to Experiments in Quantum Optics, Cambridge Univ. Press

Barbieri, C., et al. 2006, this conference

Dravins, D. 1994, ESO Messenger 78, 9

Dravins, D., Barbieri, C, Da Deppo, V., Faria, D., Fornasier, S., Fosbury, R. A. E., Lindegren, L., Naletto, G., Nilsson, R., Occhipinti, T., Tamburini, F., Uthas, H., Zampieri, L. 2005, QuantEYE. Quantum Optics Instrumentation for Astronomy, OWL Instrument Concept Study, ESO document OWL-CSR-ESO-00000-0162

Dravins, D., et al. 2006, in: T.Herbst (ed.), Instrumentation for Extremely Large Telescopes, in press (astro-ph/0511027)

Hanbury Brown, R. 1974, The Intensity Interferometer, London: Taylor \& Francis

Harwit, M. 2003, ApJ 597, 1266

Johansson, S. \& Letokhov, V. S. 2004, A\&\&A 428, 497

Johansson, S. \& Letokhov, V. S. 2005, New Astron. 10, 361

Loudon, R. 1980, Rep. Prog. Phys. 43, 913

Loudon, R. 2000, The Quantum Theory of Light, $3^{\text {rd }}$ ed., Oxford Univ.Press

Padgett, M., Courtial, J. \& Allen, L.. 2004, Phys.Today. 57, No.5, 35

Swartzlander, G.A. 2001, Opt. Lett. 26, 497 